\title{
Derivation of Sonograph Quality Parameters by the Use of Point Spread Function Analysis
}

\author{
L. DOLEŽAL, J. MAZURA, J. TESAŘÍK, H. KOLÁŘOVÁ, D. KORPAS, \\ S. BINDER, J. HÁLEK
}

Institute of Medical Biophysics, Faculty of Medicine, Palacký University in Olomouc, Czech Republic

Received May 23, 2007

Accepted May 29, 2007

On-line available May 31, 2007

\begin{abstract}
Summary
A measuring system evaluating a Point Spread Function generated in an ultrasonographic image by scanning a spherical target was developed. The target is moved in measuring bath filled by water over scanned volume via 3D computer controlled positioning system. A video signal obtained is converted to digital form and analyzed by original software to derive various objective parameters of the imager as follows: Focal areas in both the azimuth and the elevation directions, Ultrasound scanning lines visualisation, Manufacturer preloaded TGC, Width of the scanning plane, Side lobe levels and Amplification uniformity in the azimuth direction. The method was verified by testing 18 different equipments in 282 measurements. Samples of particular measurement results in form of graphical outputs are included. Medical and physiological impacts of this approach are discussed.
\end{abstract}

Key words

Ultrasonography $\bullet$ Point spread function $\bullet$ Quality $\bullet$ Imaging

\section{Introduction}

Sonographs are an important diagnostic tool in many medical applications. Their widespread application over the last ten years is due to the remarkable improvement of sonographic image quality and easy accessibility of examination. Increased importance of the examinations demands more diligent quality assessment. The first signs of ultrasonography quality assessment are observable around the year 1974, when the American Institute of Ultrasound in Medicine (AIUM) edited their first quality standards. Since then, many scientists, physicists and engineers have been active in the field
(Carson, Kollmann, Lubbers, Satrapa, Sansotta, Thijssen, Zagzebski and others). A range of quality parameters and measuring methods have been defined and developed. The International Electrotechnical Commission (IEC) is continuously preparing more precise standards for the quality assessment of sonographs. Every sonograph is equipped with a number of control functions, but these functions may complicate tasks of image quality evaluation and make the measurement more difficult. Further, subjective adjustment of the sonograph by an operator brings non-standard measuring conditions.

In addition to the quality dependence on the technology used the quality is also influenced by 
successive degradation of sonograph quality parameters in time. This is true for decay of luminescence as burning in the monitor screen is a notable reason of brightness dynamic degradation. Another common reason for stepwise loss of resolution is degradation of the transducer element's sensitivity due to piezoelectric element fatigue or mechanical damage. Frequently, damaged parts of a transducer comprise an acoustic lens and/or cable and connector. Some malfunction of electronic systems cause complete cessation of sonograph operation and cannot be abandoned by the operator. On the contrary the stepwise quality degradations, including dynamic focusing malfunctions, are not recognisable by the operator due to his continuous adaptation to the degraded picture quality. It is really a very difficult task to detect picture quality changes when imaging such complicated structures as the human body.

The stepwise degradation of picture quality is the most dangerous problem from the patient's security point of view. That's because the operator adapts to the drop of quality and missing information is substituted by his actions. Such a situation leads to a wrong diagnosis. Therefore a routine periodical sonograph imaging quality assessment is vitally important to ensure safety of both, the patient and the sonographer.

The sonograph image quality is determined by the following parameters: Spatial resolution, Contrast resolution, Temporary (time) resolution, Dynamic range, Gray scale steps (the AD converter range), Sensitivity (Maximum depth imaged), Transmitted energy intensity level, Side-lobes level, Signal-to-noise ratio, Dead zone range, imaging monitor dynamic range and spatial resolution (the PIXEL size). Most of the imaging quality assessment methods are based on an observer's subjective evaluation of the sonogram (ROWND 1997). These methods are not acceptable for an objective decision process. A trend to find sensitive, precise and objective methods has led to the development of two groups of measuring methods. The first method contains fast and easy to operate routine tests, dedicated to periodical screening of quality parameters directly in sonographic examination rooms (Barnett 2000, Kollmann 2003, Kollmann 2005). The second group is formed from more accurate and more sophisticated methods, requiring well skilled operators and mostly being available in special laboratories only (Szabo 2004).

We have developed a measuring system which belongs to the second group of methods. The system evaluates a Point Spread Function which is generated in an ultrasonographic image by scanning a spherical target.

\section{Method}

As explained, the basic component of the system is a spherical point reflector, which is made of metal, fixed by suitable holder and positioned in a measuring bath in axial, azimuth and elevation direction by a $3 \mathrm{D}$ computer driven positioning system. The computer is used not only to control the target position but it manipulates input image data, analyses the measured data and arranges the form of the output results. The Diameter D of the reflector is not very critical, but should be kept within a range $\lambda<\mathbf{D}<\mathbf{4} \lambda$ (Doležal 2005).

\section{Measuring conditions}

Every sonograph is equipped with a large set of different control functions to ensure good optimal conditions for the manipulation of received ultrasound signals to create the best image. Because the method evaluates the signal after it has passed through a whole imaging system all the functions affect the measurement results. Therefore the controls must be reproducible. All the adjustments and settings must therefore be carefully reported and recorded.

Step 1 is the amplitude transfer characteristic adjustment:

To encode a very wide range of received signal amplitudes the sonograph receiver amplifier has to have a logarithmic transfer characteristic. Therefore all other additional nonlinear, so called pre-processing functions, have to be switched off or adjusted to their linear working regions. This includes functions dedicated to eliminating noise either by scan sequences correlation or averaging or cutting off low level signals.

The second step is the measuring system dynamic range adjustment:

The amplified signal peak amplitude limitation, due to low amplifier dynamic range, must not be used to assure measurement of the FWHM (Full Width in Half of the Maximum) function. Therefore the widest dynamic range accessible by the measuring system has to be adjusted.

The third condition concerns system gain adjustment:

The sonograph's receiver gain is controlled in two parts, the first is the General Gain (GG) and the second one is the Time Gain Compensation (TGC) function. Their adjustment prior to measurement should 
be as follows: The TGC is adjusted first. The basic condition is to adjust the TGC constant within the whole of the scanned depth. The level of the TGC is defined at the middle of its range. In some cases another level should be used due to the TGC influence to the general gain. The main condition of the TGC adjustment is its independence from the depth parameter (elimination of the function).

Then the GG is adjusted to just obtain rather visible noise on the screen.

The fourth step - the look up table setting:

The function of the grey scale level to the digitalised amplitude of the signal is derived from a Look-Up-Table (LUT) or Gamma curve. To assure a pure logarithmic characteristic of the system a linear LUT should only be used.

All the settings are documented in the measuring protocol to be recorded for use by further periodical measurements.

\section{The data acquisition system}

The system grabs a continuously recorded video signal from the sonograph. Prior to running the acquisition a proper Region of Interest (ROI) is chosen in the sonogram. The software controls ROI position according to the target replacement in scanning plane to keep the target inside the ROI. Only that ROI frames are used for further analysis, which coincides with the target position in the pre-programmed measuring grid points within the measured volume of the scan. Each of these ROIs is analyzed to find its highest PIXEL value when the target moves in an elevation direction. Not whole ROIs but their highest PIXEL values are stored including corresponding target position for each elevation path. Only one ROI frame from each elevation set is stored. It contains maximum from all the highest PIXELs. The stored ROI is a picture of target in centre (axis) of the ultrasound beam and therefore may be analyzed to find the PSF in both the azimuth and the axial directions.

The input data are yielded from a composite video signal. That's why the video signal level and the transfer characteristic may affect the results and thus have to be well adjusted to assure use of the whole range of the AD converter to prevent artificial limitation of the high level signal. To avoid such malfunctions a software controlled frame grabber NI PCI-1411®) (National Instruments) is used. Its sensitivity can be adapted to the video signal level by use of National Instruments software NIMAQTM, which is a part of the Lab View ${ }^{\mathrm{TM}}$ developing system.

\section{Results}

The work has given the following results on the base of the ROI and target position dependent data analysis over the scanning area:

The ROI stored for each scanned plane axis in each point of the measuring grid.

The echo signal amplitude distribution over the measured area.

Distribution of the azimuth Point Spread Function (PSF) FWHM over the measured area.

The peak echo amplitude at each step of the target position in the elevation (transversal) direction.

Using these data, we are able to derive the following ultrasound scanner parameters:

1. Focal areas in both the azimuth and the elevation directions

2. Ultrasound scanning lines visualisation

3. Manufacturer preloaded TGC

4. Width (elevation) of the scanning plane

5. Side lobe levels

6. Amplification uniformity in the azimuthal direction.

Focal areas in both the azimuth and the elevation directions

The focal area position is characterised by spatial resolution and sensitivity limit. Therefore the focus position is derivable from the FWHM of PSF distribution over the corresponding direction as seen in Fig. 1A. In the case of dynamic focussing not only the position but the number of focuses is available to enable analysis from the characteristic (see Fig.1B).

\section{Ultrasound scanning lines visualisation}

When the elevation step of the ball target replacement in lateral direction is less than $1 / 4$ of the ultrasound line space, fluctuation of received signal amplitude is observable along the scanning lines. This method may be used to visualize separate scan lines, their density and changes of profile (as Fig. 2 shows).

\section{Preloaded TGC}

When the TGC is adjusted to be depth independent we can derive the manufacturer preloaded TGC function from the received signal amplitude level distribution in the axial profile (Fig. 3). The preloaded 
A.

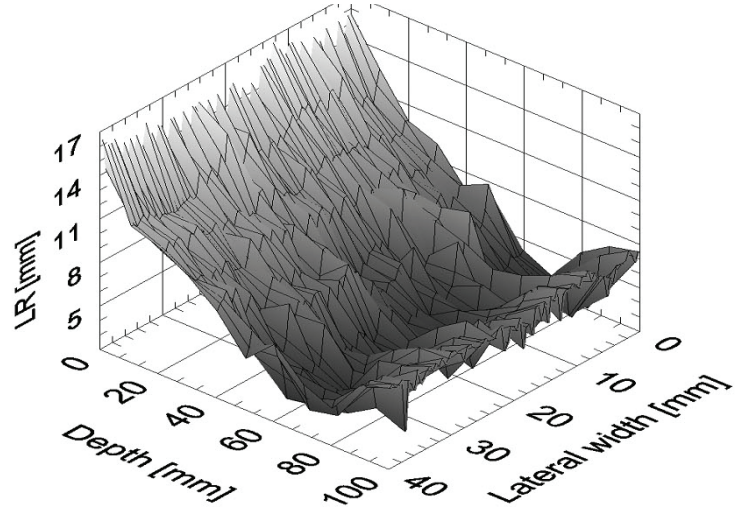

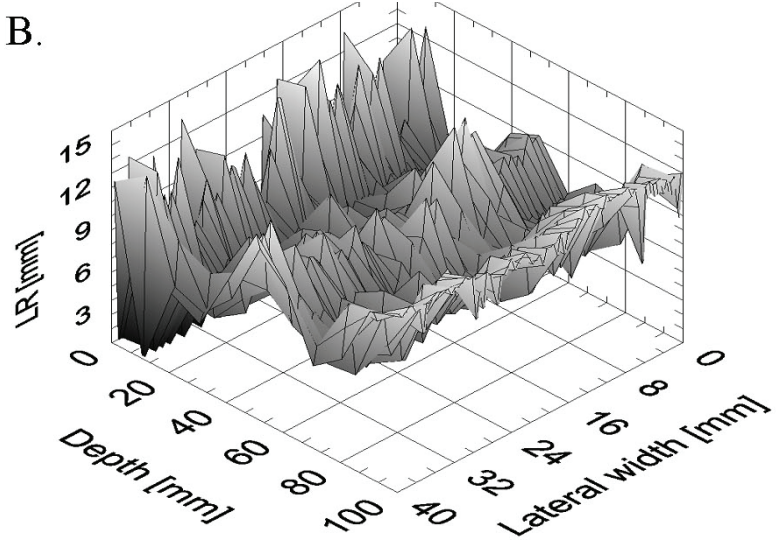

Fig. 1. Dynamic focuses number and position may be detected from FWHM characteristic shape. The lowest value corresponds with focus position. The A characteristic detects one focal point in depth about $70 \mathrm{~mm}$. The characteristic B detects two focal points, positioned in $30 \mathrm{~mm}$ and $70 \mathrm{~mm}$ depth respective. Improvement of azimuth resolution close to the transducer as a result of second focus application is evident from characteristic B. The measured transducer was linear, $3.5 \mathrm{MHz}$. The depth distance value in relation to measured area, not to the distance from transducer. Remark: The FWHM axis is labeled as LR (lateral resolution).

A.

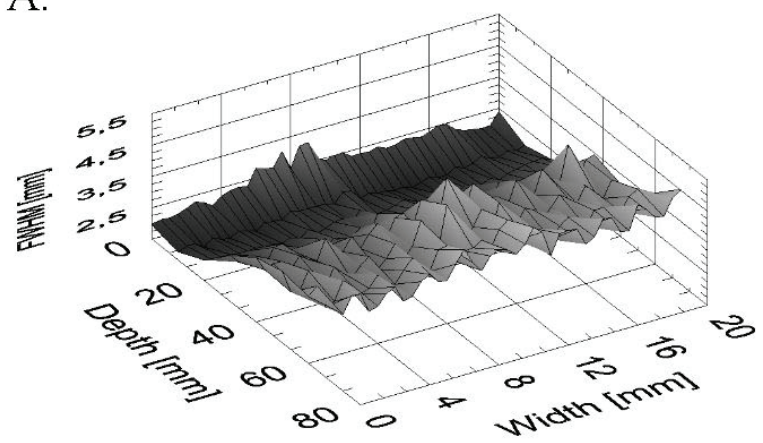

B.

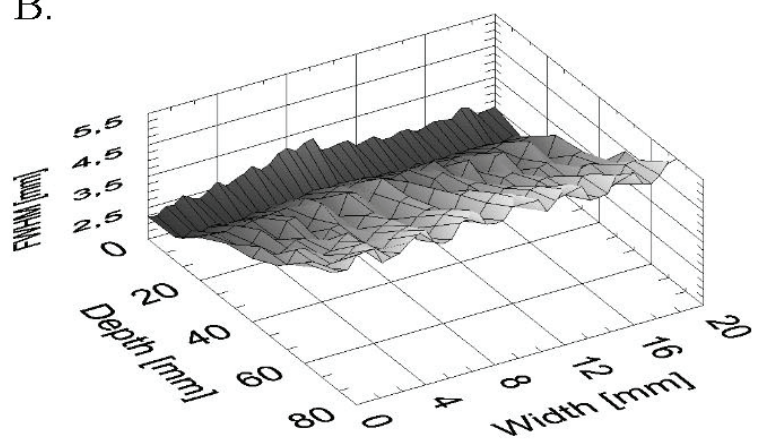

Fig. 2. Number and therefore density of ultrasound scan lines can be derived from the FWHM or the brightness characteristics. In case of the first one the lover value indicates centre of ultrasound scan line. When the brightness characteristic is concerned, the criterion is reverse - maximum will be the centre of scan line. Characteristics $\mathbf{A}$ and $\mathbf{B}$ were recorded with $2 \mathrm{MHz}$ phased array transducer. Two different line density modes (high and low) were adjustable for the transducer. The characteristic $\mathbf{A}$ was recorded using high density mode and the second one, the $\mathbf{B}$, with low density mode. The first case gives 10 lines per $20 \mathrm{~mm}$ and the second case 7 lines per $20 \mathrm{~mm}$ width of scan. There is shift of focal area observable between the A and B characteristics. The B case uses dynamic focusing more close to the transducer.

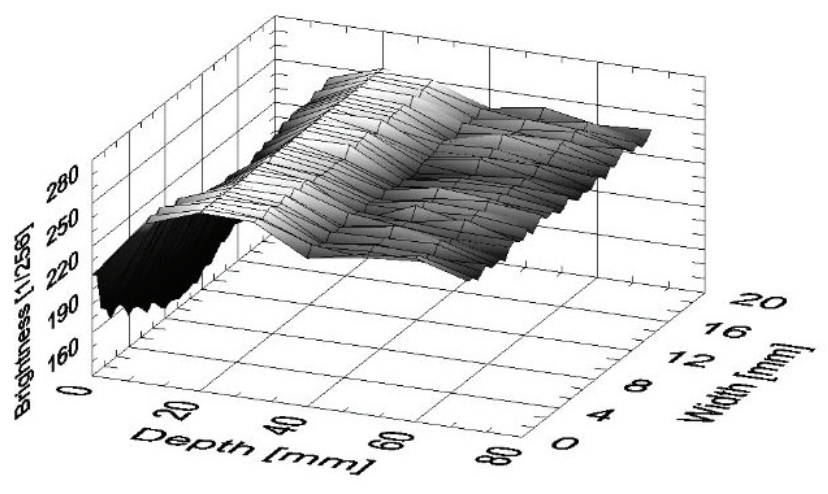

Fig. 3. The echo amplitude distribution is carrying information about the gain distribution. The scanned area is laying in the measuring bath filled in by degassed water. For constant gain a constant level of peak received signal is assumed to be distributed over scanned area because ultrasound energy absorption in the water is negligible. This graph informs us about manufacturer presented attenuation in a near field of transducer. The vertical axis is calibrated in steps of 8 bit PIXEL. The peak wave in depth $20 \mathrm{~mm}$ in this graph corresponds with position of the first azimuth focal point of measured phased array matrix sector scan transducer working on frequency 2 $\mathrm{MHz}$. 
TGC curve is very often preprogrammed in the sonograph software and used transducer dependent. An aim of this function is to reduce sensitivity for the image area close to the transducer.

\section{Elevation width of a scanning plane}

This is assessable from the transversal profile of amplitude in a specified point of the measuring grid. There is an informative graph in the program imaging window chosen characteristic registered on the calibration sonogram. Use of this window is called "browsing" because in this form we can display measured and calculated values including particular ROIs at any measured point of the grid by "browsing" the characteristic using a cursor. Fig. 4 shows 6 elevation profiles, analyzed in depths between 5 and $15 \mathrm{~cm}$ in step of $2 \mathrm{~cm}$.

\section{The Influence of side lobes on imaging quality}

It is highly significant for electronic transducers characterized by a high ratio between thickness and length of transducer element. The side lobes are responsible for high noise and speckle levels and spatial interference artefacts and ghosts in the sonogram generated. The elevation (transversal) side lobes are readable as the transversal amplitude profile displayed in the "browser" window as shown in Figure 4. The azimuth side lobes level is estimated in the ROI by reading the values stored in spherical reflector side lobes reflection PIXELs (Fig. 5).

\section{Amplification uniformity in the azimuthal direction}

The 2D sonogram is composed of individual scan lines which fill the scanning plane in the azimuth direction. The input analogue signal is evaluated in groups of lines by sets of analogue channel amplifiers. The channel characteristics should be uniform to avoid brightness irregularities in the picture. Using the point target echo amplitude distribution function can emphasise such irregularities because they form periodically repeated changes of the echo intensity corresponding to a certain number of scan lines (the channel set width) as
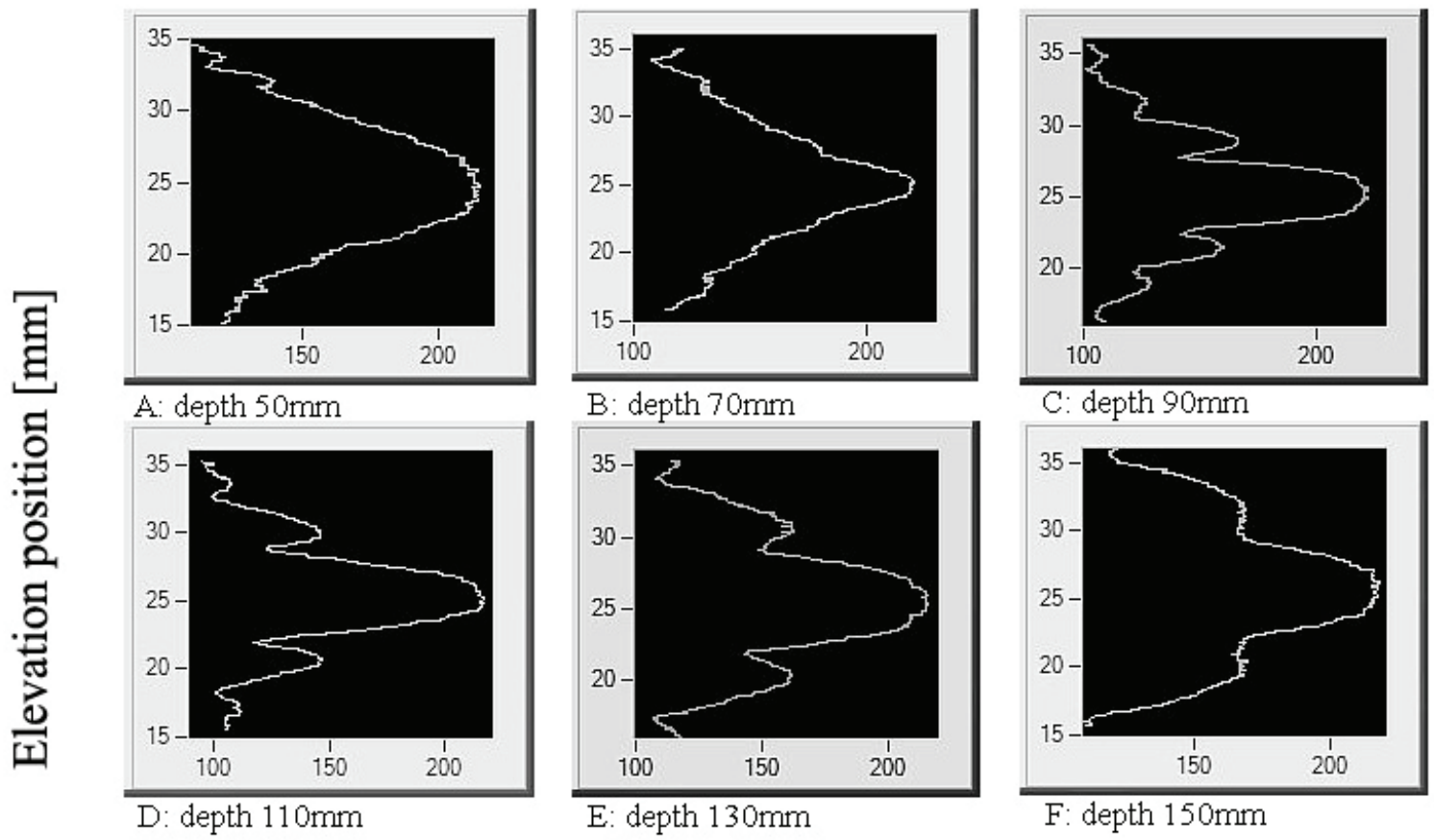

\section{Pixel maximum level in ROI}

Fig. 4. Estimation of the focal area in elevation plane and elevation thickness of the scan. Measured transducer was a matrix convex type, frequency $5 \mathrm{MHz}$. Transversal focal area could be expected from the narrowest curve in depth about $7 \mathrm{~cm}$ (picture B). Slices $\mathrm{C}-\mathrm{F}$ show elevation side lobes in different depths in step of $2 \mathrm{~cm}$. The distance is measured from surface of the transducer. Vertical axis is labeled in $\mathrm{mm}$ of elevation displacement of target and horizontal axis in digitalization steps (the range from 0 till 255). 


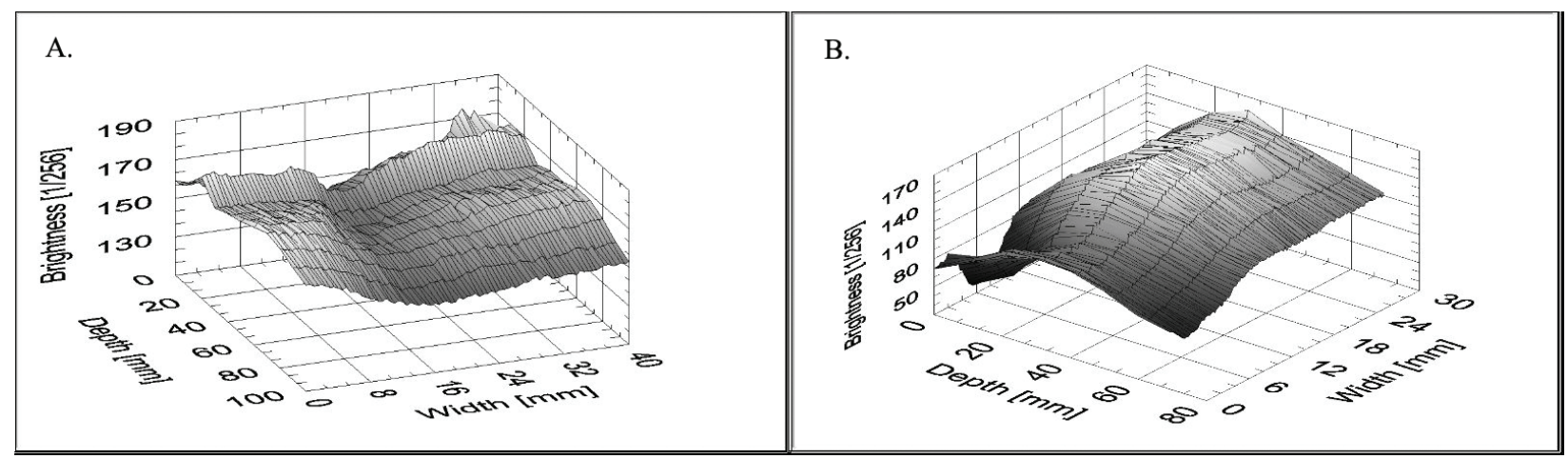

Fig. 6. The echo amplitude distribution depends on transducer sensitivity in particular area and corresponding amplifier channels parameters. The Fig. 6A performs characteristic of phased array sector transducer where failure in beamformer could be expected (all crystals participate on generating each scan-line). The Fig. 6B introduces linear transducer characteristic with damage of acoustic lens between 0 and $12 \mathrm{~mm}$ of its evaluated part.

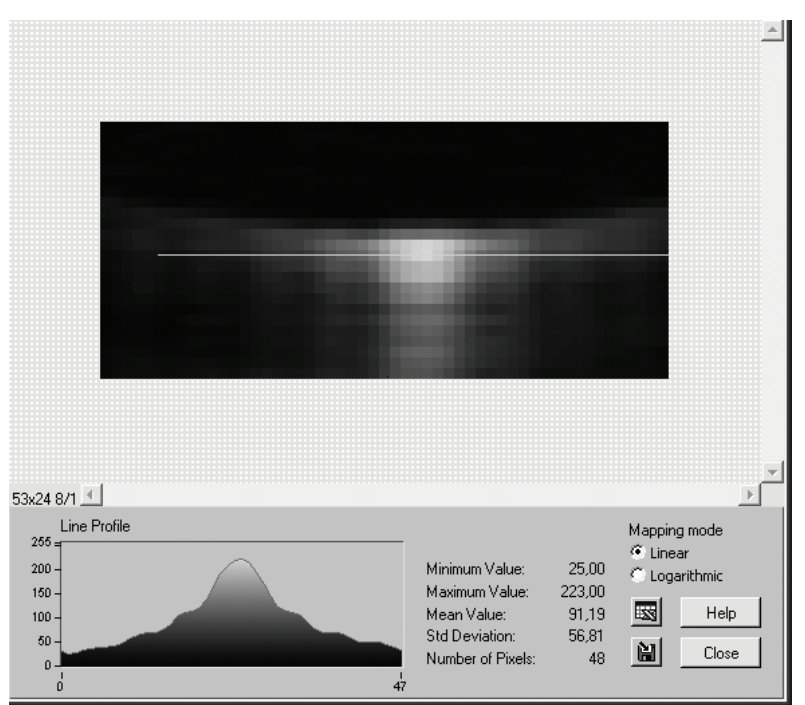

Fig. 5. Azimuth plane side lobes level assessment is provided using National Instrument NIMAQ ${ }^{\text {TM }}$ software. The amplitude level of side lobes is measured in the area of ROI. The side lobes generate "umbrella shaped" structures symmetric toward the centre of target (the pixel containing highest number) in the image.

seen in Figure 6A. Although malfunction or partial malfunction of several crystals in transducer is easy to detect using this information (Fig. 6B).

We have been developing the method of PSF analysis for more than 10 years. During the last three years, we have been able to analyze all the listed parameters using the last model of the measuring system prototype and program analysis. In total 282 measurements have been performed in the period between February 2004 and September 2006. We have analyzed 18 different sonographs using 52 different transducers of all the most conventional types (linear, convex, mechanical, and/or phased array sector). All the results obtained confirm our expectations to yield comprehensive and accurate information about the parameters listed above.

\section{Medical and physiological impact of the results obtained}

Ultrasonography is a diagnostic technique used to detect pathology, which exhibits as changes in tissue morphology. These microscopic changes present a challenge for ultrasound spatial resolution. Examples of such critical ultrasound examinations are, vessel wall imaging to detect changes of structure and sclerotic plaques origin; observing ductus choledochus lumen, pancreatic structures; changes of the tissue structures in mammary or thyroid glands; foetal abnormalities and foetal echocardiography; uterine and ovarian malformations etc.

These examinations demand high quality imaging methods to be of maximum clinical benefit. As is discussed - our method is able to measure some important quality parameters, e.g. individual adjustment of the scanning focal area. The effect of incorrect adjustment of the focus to the sonogram quality is illustrated in Fig. 7A, B, where influence of the focal area position in thyroid gland sonogram is displayed.

Some research still continues to find evidence of undesirable physiological side effects due to ultrasound irradiation. Even if this is not proven, the rule of ALARA (As Low As Reasonably Achievable) is still recommended. Consequently - a reduction of the examination time without a reduction in diagnosis quality is desirable using sonograph with better resolution. 

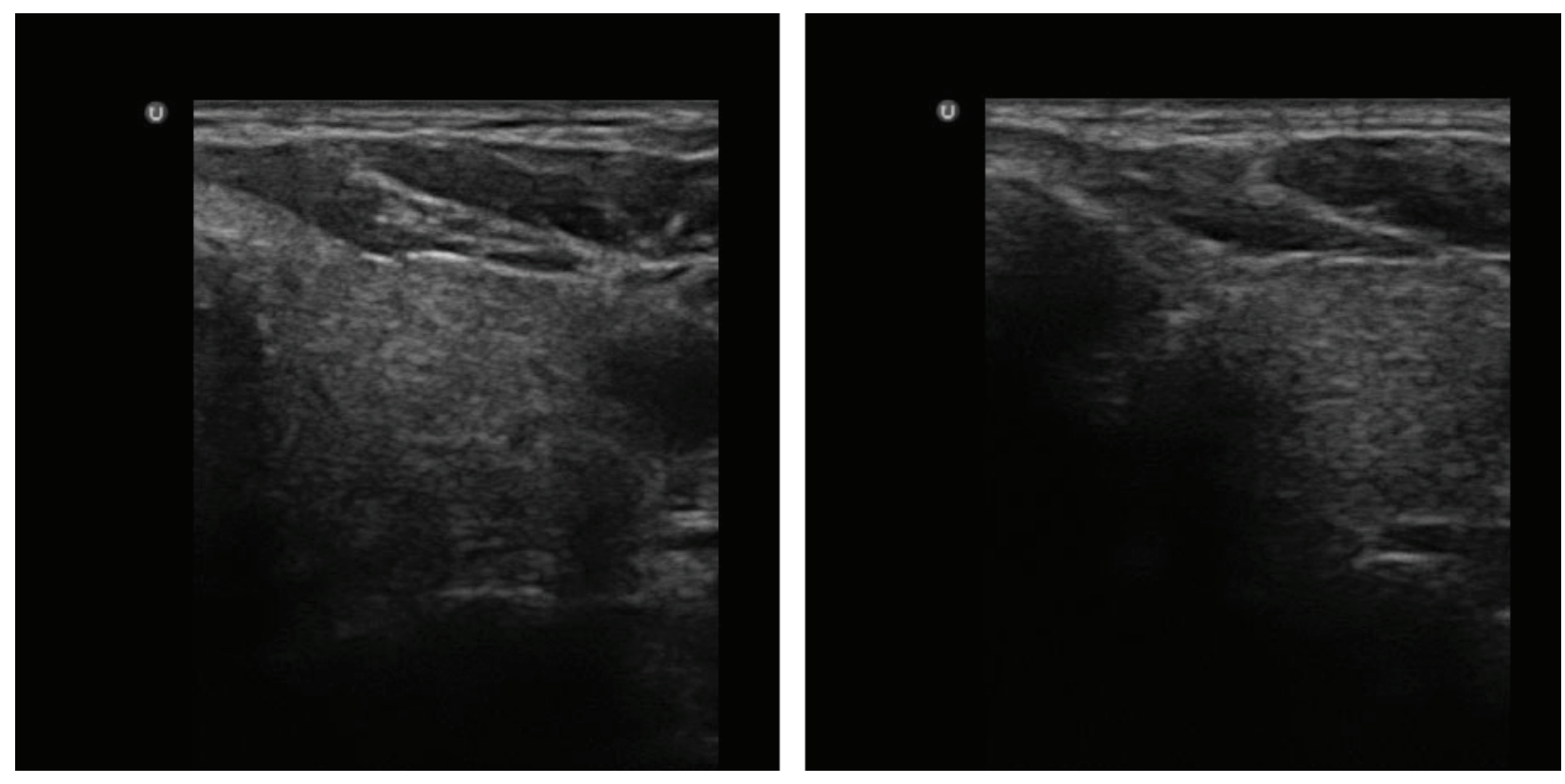

Fig. 7. The lateral dynamic focusing effect is documented on sonogram of thyroid. The left hand side picture was scanned using properly functioning focusing while the right one with focusing being not correct. Some details are missing and sensitivity is lower.

\section{Discussion}

We have found that our measuring equipment gives sufficiently accurate and objective results. We have the ability to detect different parameters, using original methods, to derive specific comprehensive information. Some results are original, and are not obtainable from other methods, e.g. evaluation of received signal level distribution, channel uniformity, focus and focal area specification also side lobes specification.

The draw-back of the method is high time consumption depended on size and spacing of the measuring grid and demand of high skilled operator.

The method may be used for laboratory evaluation of different scanners to specify or check their parameters. A further field of application is research and development of ultrasonographs to examine new technical aspects of construction.

\section{Acknowledgements}

Authors would like express their gratitude to the Czech Ministery of Education, Youth and Sport for financial support by grants: "KONTAKT 1P2004 ME720" and MSM 6198959216.

Important support was received from Mrs. Terri Gill, who kindly and effectively corrected our English.

\section{References}

BARNETT SB, TER HAAR GR, ZISKIN MC, ROTT HD, DUCK FA, MAEDA K: International recommendations and guidelines for the safe use of diagnostic ultrasound in medicine. Ultrasound in Med. \& Biol 26: 355 - 366 , 2000.

DOLEŽAL L, KOLLMANN CH, HÁLEK J: Ball target diameter impacts the Measured Sonograph Point Spread Function. Scripta Medica 78: 211 - 218, 2005.

IEC 61391- 1: Ultrasonics - Pulse echo scanners - Part 1 Ed.1.0: Techniques for calibrating spatial measurement systems and measurement of system point spread function response. International Electrotechnic Commission, Geneva: 2006.

IEC 61391 - 2: Ultrasonics - Pulse echo scanners - Part 2 Ed.1.0: Techniques for measurement of maximum depth of visualisation and the displayed dynamic range. International Electrotechnical Commission, Geneva: preliminary info, expected year of application 2010. 
KOLLMANN CH: Aspects and practical concepts for performing Quality Assurance of Ultrasound imaging equipment in clinical use. Proceedings Patient Ultrasound Interaction; Palacky University Olomouc: 4-7, 13.6.2003.

KOLLMANN CH: Quality Assurance of Ultrasound equipment - Austrian Test kit, Type Basic (Vers.1, 2005), white paper, ZBMTP, www.meduniwien.ac.at/zbmtp/people/kollch1/download/download.html.

LUBBERS J, GRAAFF R: Flat Ended Steel Wires, Backscattering Targets for Calibrating over a Large Dynamic Range. Ultrasound in Med \& Biol 32: 1585-1599, 2006.

ROWND J J, MADSEN E L, ZAGZEBSKI J A, FRANK G R, DONG F: Phantoms and automated system for testing the resolution of ultrasound scanners. Ultrasound in Med \& Biol 23: 245-260, 1997.

SZABO TL: Ultrasonic exposimetry and acoustic measurements. In: Diagnostic ultrasound imaging - inside out. TL SZABO (editor), Elsevier Academic Press, London, 2004, pp 429-453.

THIJSSEN JM, WEIJERS G, KORTE CL: Objective Performance Testing and Quality Assurance of Medical Ultrasound Equipment. Ultrasound in Med \& Biol 33: 460-471, 2007.

\section{Corresponding author}

Ladislav Doležal, Ústav lékařské biofyziky, Lékařská fakulta UP, Hněvotínská 3, 77515 Olomouc. E-mail: ladol@tunw.upol.cz 